ArtefaCToS. Revista de estudios de la ciencia y la tecnología

eISSN: $1989-3612$

Vol. 7, No. 1 (2018), 2a Época, 51-73

DOI: http://dx.doi.org/10.14201/art2018715173

\title{
La relevancia de los recursos cognitivos en un entrenamiento de natación sincronizada: El caso de andamiajes y affordances ${ }^{\dagger}$
}

\section{The Relevance of Cognitive Resources in Synchronized Swimming Trainings: The Case of Scaffoldings and Affordances}

\section{Dafne MUNTANYOLA-SAURA*; David CASACUBERTA**;} Anna ESTANY***

${ }^{*}$ Universitat Autònoma de Barcelona, España

Centre d'Estudis Sociològics sobre la Vida Quotidiana i el Treball (QUIT) Institut d'Estudis del Treball (IET)

dafne.muntanyola@uab.cat

${ }^{* *}$ Universitat Autònoma de Barcelona, Departament de Filosofia, España david.casacuberta@uab.cat

${ }^{* * *}$ Universitat Autònoma de Barcelona, Departament de Filosofia, España anna.estany@uab.cat

Recibido: 10/01/2018. Revisado: 18/01/2018. Aceptado: 23/01/2018

\section{Resumen}

En el entrenamiento de natación sincronizada confluyen elementos artísticos y deportivos de alta competitividad. Nos preguntamos cómo contribuyen los andamiajes y affordances a la estabilización de los recursos cog-

\footnotetext{
† Este trabajo se enmarca en el proyecto del Ministerio de Ciencia e Innovación de España "Creatividad, revoluciones e innovación en los procesos de cambio científico". (Referencia FFI201452214-P).

Agradecemos a Anna Tarrés, entrenadora del equipo olímpico español de natación sincronizada, a su colaboradora Beth Fernández y a las nadadoras que obtuvieron la medalla de plata en el duo formado por Andrea Fuentes y Ona Carbonell y la medalla de bronce al conjunto del equipo en los Juegos Olímpicos de Londres 2012, por su disponibilidad para realizar este trabajo. También al Centro de Alto Rendimiento (CAR) por las facilidades en poder observar los entrenamientos.
} 
nitivos de la entrenadora y de las nadadoras del equipo olímpico español de natación sincronizada. Vamos a detenernos en qué se entiende por recursos cognitivos y en los posibles sentidos de andamiaje y affordance. $Y$ examinamos las pautas comunicativas y el proceso de trabajo que da lugar al entrenamiento de natación sincronizada. Proponemos una etnografía cognitiva de los entrenamientos, un método innovador que sistematiza los patrones de actividad de las interacciones locales entre los participantes de la formación deportiva. Hemos utilizado el programa ELAN e incorporado las técnicas de descripción jeffersonianas que provienen del método de análisis conversacional. Los resultados muestran cómo el proceso de entrenamiento implica la interacción en un entorno que incluye otros agentes sociales, recursos materiales y modelos conceptuales que se retroalimentan.

Palabras clave: entrenamiento; etnografía cognitiva; multimodalidad; movimiento.

\begin{abstract}
In synchronized swimming, highly competitive trainings arts and sports elements converge. We wonder how scaffoldings and affordances contribute to the stabilization of cognitive resources among the trainer and swimmers of the Spanish Olympic synchronized swimming team. We dwell on what is taken as cognitive resources and the role of scaffolding and affordances. We analyze communication patterns and examine the work process leading to synchronized swimming. We propose a cognitive ethnography on training, an innovative method that explains the patterns of activity of local interactions among participants in sports. We applied ELAN program and incorporated Jeffersonian transcriptions that come from conversational analysis method. Results show how the training process involves interaction in an environment that includes social agents, material resources and conceptual models that feed each other.
\end{abstract}

Keywords: Training; Cognitive Ethnography; Multimodality; Movement.

\title{
Introducción
}

En el entrenamiento de natación sincronizada en la que confluyen recursos conceptuales, materiales y sociales, entrelazados pero distinguibles, los recursos cognitivos son claves para cualquier actividad humana, desde las más simples hasta las más complejas, y desde las más cotidianas a las más especializadas, sean estas científicas, artísticas, deportivas, etc. Por tanto, no es de extrañar que una actividad como la natación sincronizada en la que confluyen elementos artísticos y deportivos de alta competitividad sean relevantes los recursos cognitivos y, 
muy especialmente, los scaffoldings (andamiajes) y las affordances. "No somos dioses", aunque esta frase es una perogrullada, no está de más tenerla en cuenta a la hora de abordar las posibilidades y límites de la naturaleza humana. Podríamos referirnos a una gran variedad de situaciones en las que estas posibilidades y límites se hacen evidentes y también de la infinidad de recursos para superarlas.

El objetivo del artículo es analizar algunos de los recursos cognitivos más relevantes para la natación sincronizada, poniendo especial énfasis en andamiaje y affordance. En primer lugar, vamos a detenernos en qué se entiende por recursos cognitivos y en los posibles sentidos de andamiaje y affordance. En segundo lugar, examinaremos "las pautas comunicativas que tienen lugar en los entrenamientos de natación sincronizada del equipo olímpico español” (Muntanyola-Saura, 2015). Al mismo tiempo analizaremos "el proceso de trabajo que supone la natación al mantener los hechos tal y como suceden en que se sitúan las implicaciones cognitivas y la pertinencia social" (Muntanyola-Saura, 2015). Finalmente, nos preguntamos cómo contribuyen los andamiajes y affordances a la estabilización de los recursos cognitivos de la entrenadora y de las nadadoras del equipo olímpico de natación sincronizada.

Estudios recientes de claro cariz cognitivo van en la dirección de la "corporeidad" de la mente (o de la mente "corpórea") o con la expresión de conocimiento socialmente distribuido (Gibbs, 2006; Hollan, Hutchins y Kirsh, 2000). Los recursos cognitivos dentro de esta perspectiva no son un componente o instancia exclusivamente interna, sino que se externaliza en los procesos de trabajo: en las pantallas, protocolos, conversación, resultados, etc. De esta manera, los recursos cognitivos parecen ser andamiajes y affordances compartidas como objeto de acuerdo (o desacuerdo) entre los agentes intervinientes. Se ha de tener en consideración la importancia de la intervención de los artefactos en los procesos de trabajo. En este sentido, no se puede establecer una dicotomía entre acciones comunicativas, cognitivas y acciones instrumentales a partir de la distinción entre agentes humanos y los instrumentos de trabajo. Toda práctica interactiva proviene también y está mediatizada por los artefactos, máxime en los procesos de trabajo actuales: sistemas notacionales, automatización, mecanismos de información y cálculo, robotización, tecnologías cada vez más "inteligentes”, que también están presentes en la preparación de los entrenamientos de natación sincronizada (Hutchins, 1995; Hutchins y Klausen, 1996; Lozares, 2001, 2007). Este enfoque tiene un gran valor añadido: explicando cómo se producen los mecanismos y procesos cognitivos (intencionales, representaciones, estrategias) y

\footnotetext{
${ }^{1}$ Los términos "scaffolding" y "affordance" son muy comunes en ciencia cognitiva, y no hay acuerdos para su traducción. Consideramos que "andamiaje" captura muy bien los conceptos y aplicaciones detrás de "scaffolding" y así lo dejaremos en este texto. "Affordance" por el contrario, es un término técnico del psicólogo Gibson a partir del verbo "to afford" que no permite una traducción elegante al castellano. "Permitir" o "Proporcionar" no tienen mecanismos sencillos para convertirse en nombres y "funcionamientos" es demasiado genérico. Ver en Lozares (ed.) (2007) la nota de la traductora sobre el término affordance en español.
} 
resultados en entornos locales o situacionales desvela la caja negra de la psicología folk, y permite una explicación más integrada de la acción (Lozares, 2007; Muntanyola-Saura, 2014). La antropología cognitiva (D’ Andrade, 1995; Hutchins, 2005) continúa la tradición durkhemiana de buscar una ontología de categorías cognitivas en los patrones relacionales y estructurales. En un marco weberiano, la acción es importante como parte de las tradiciones pragmáticas interactivas tales como la etnometodología (Garfinkel, 1967) la interacción simbólica (Goffman, 1961), la sociología cognitiva (Cicourel, 2012) y la etnografía (Knorr- Cetina, 1999; Wacquant, 2004).

Para analizar y localizar los andamiajes y affordances en tanto que recursos cognitivos comunicativos, proponemos una etnografía cognitiva de los entrenamientos de natación sincronizada (Williams, 2004). Se trata de un método naturalista basado en la observación y las entrevistas que sistematiza los patrones de actividad de las interacciones locales entre los participantes de la formación deportiva. En líneas generales se siguen los principios de la "grounded theory" (Corbin y Strauss, 1990) que mantiene el esquema de interpretación circular de los datos etnográficos. Como herramienta hemos utilizado el programa ELAN. Como innovación, hemos incorporado las técnicas de descripción jeffersonianas que provienen del método de análisis conversacional (Sacks et al., 1978). Se trata de una forma de transcripción que nos ha permitido entrar en el detalle de la interacción, no sólo a un nivel puramente lingüístico, sino a nivel multimodal, tomando en cuenta gestos, acciones corporales y otros elementos no verbales de comunicación.

\section{Marco teórico de la investigación}

\section{1. Génesis y polisemia de andamiajes y affordances}

Un panorama exhaustivo de los sentidos de andamiaje y affordance va más allá de los objetivos de este trabajo. Lo que nos proponemos es ejemplificar la polisemia de este concepto y hacer ver sus raíces en los conceptos de andamiaje y affordance. A pesar de que a continuación dedicamos un apartado a cada uno de los conceptos, no partimos de una diferenciación establecida de antemano, sino que se trata de tomar uno de estos conceptos como punto de referencia, ver los diversos sentidos del mismo y, al mismo tiempo, analizar las posibles diferencias y semejanzas con el otro concepto.

La idea de andamiaje procede del psicólogo ruso Lev S. Vygotsky y tiene el sentido de la ayuda que los adultos proporcionan a los nińos para aprender y desarrollar habilidades cognitivas complejas. Relacionado con la idea de andamiaje está lo que Vygotsky (1978) llama "zona de desarrollo próximo" (zone of proximal development), refiriéndose a la brecha que hay entre lo que un niño 
puede alcanzar solo y lo que puede alcanzar con la ayuda, bien de los adultos bien de la colaboración con otros niños, una idea que no puede separarse del enfoque sociocultural del desarrollo.

En la línea de Vygotsky encontramos en 1976 a Wood, Bruner y Ross (1976) que introducen el término andamiaje para describir la interacción tutorial entre un adulto y un niño, un sentido muy parecido al de Vygotsky. También Dunlap y Grabinger (1996, 242) utilizan el término andamiaje para referirse al apoyo de los educadores a los niños a través de guías adecuadas a la edad y a su nivel de experiencia. Estas guías son predominantemente conceptuales, aunque se utilicen elementos materiales como soporte para que puedan llevarse a cabo. Todas estas referencias tienen un denominador común que es el de la educación y el aprendizaje, en que los adultos (u otros nińos, que se supone que ya han aprendido la lección y, por tanto, son más expertos) facilitan estos andamiajes.

La aplicación a la educación tendría su equivalente en la práctica científica en facilitar las tareas al aprendiz o becario por parte del experto o director del laboratorio. Precisamente en este sentido es relevante la utilización de Hutchins, refiriéndose a andamiajes como lo que facilita al novicio el aprendizaje de lo que en la navegación llama "guardia en cierta clase de barcos" (Standard Steaming Watch). Hutchins $(1995,280)$ señala que "el andamiaje proporcionado al novicio por los otros miembros de equipo está construido sobre lo que culturalmente se considera duro o fácil de aprender”. Por tanto, la referencia de Hutchins está muy en consonancia con la primera idea de Vygotsky, relacionada con el aprendizaje y con el acento puesto en los modelos culturales.

Otro de los autores actuales que se refieren a andamiajes es Clark (2004), quien analiza diferentes sentidos de este concepto. Entre los más relevantes para la práctica científica podemos señalar los siguientes:

i. El andamiaje como instrumento conceptual; en este sentido el problema cuerpo/mente sería un andamiaje que nos ayudaría a comprender cómo el pensamiento y la razón humana surgen de la interacción entre cerebros materiales, cuerpos materiales y entornos culturales y tecnológicos (Clark, 2004,11).

ii. El andamiaje como ayuda crucial para el cerebro que, aunque potente, tiene sus limitaciones; en la misma línea está la idea de "pensar con andamios" (scaffolded thinking).

iii. El lenguaje como andamiaje que nos permite convertir nuestros propios pensamientos en objetos de reflexión. Cuando "congelamos" un pensamiento en palabras lo convertimos en objeto de análisis (Clark, 2004, 79). 
El sentido i) sería una forma de andamiaje conceptual, de hecho, él mismo lo denomina "instrumento conceptual", lo cual implica que forma parte de algún modelo cultural, que pueden utilizar los que lo comparten. El sentido ii), por un lado, parece el que más en consonancia está con la idea original de Vigotsky, es decir, el andamiaje nos proporciona un recurso para aumentar nuestra capacidad cognitiva; por otro, hay una diferencia y es que Vigotsky piensa en un contexto educativo y en que quien proporciona el recurso son los padres y educadores en general, mientras que, en Clark, el recurso con el que piensa, fundamentalmente, es la tecnología o cualquier artefacto material como un bloc de notas o las tablas de sumar. Respecto al lenguaje como andamiaje, tal como lo toma Clark, parece que lo focaliza en la dimensión material en tanto en cuanto lo interpreta como una forma de "congelar" los pensamientos. Respecto al lenguaje como andamiaje es pertinente lo que Vigotsky señala sobre la relación entre lenguaje y pensamiento: "La relación del pensamiento con la palabra no es una cosa sino un proceso, un movimiento continuo de ida y vuelta del pensamiento a la palabra y de la palabra al pensamiento" (Vygotsky, 1962, 125).

Dentro de la evolución del tratamiento de andamiaje el estudio de Mascolo (2005), amplía y clasifica dicho concepto más allá de su concepción original. Mascolo utiliza la expresión "andamiaje interactivo" (coactive scaffolding) como el recurso cognitivo que ocurre "cuando elementos del sistema persona-entorno que se encuentran fuera del control directo de un actor individual dirige o canaliza la construcción de la acción de formas nuevas y no anticipadas" (Mascolo, 2005, 187). Por tanto, la clasificación es de los diferentes andamiajes interactivos, no de andamiajes sin calificativos.

Así distingue entre andamiaje social, ecológico y auto-andamiaje (Social, Ecological y Self-scaffolding). El social es el que más se adecuaría a la idea de Vygots$\mathrm{ky}$, en tanto en cuanto lo relaciona con procesos mediante los cuales se producen intercambios con otras personas, sean entre educador y nińo, experto y novicio, o entrenador y jugador. En cuanto al ecológico, M.F. Mascolo lo define en los términos siguientes: "se refiere a la manera en la que las relaciones o posiciones de uno dentro de una ecología física y social amplia mueve la acción hacia formas noveles". "Auto-andamiaje", se trata de que las acciones del propio individuo crean nuevas condiciones para nuevas formas de acción y de significado. Uno de sus ejemplos es el del juego del 'Scrabble' en que la acción del individuo al mover las fichas cambia las condiciones y proporciona nuevas formas de acción. En este sentido podemos verlo como andamiaje en el sentido de Vygotsky, pero en lugar de ser otros (los educadores) los que proporcionan recursos, son los propios individuos los que se los proporcionan a sí mismos. Así, en un experimento también sobre Scrabble diseñado por Maglio y Kirsh (Maglio et al., 1999), la hipótesis de trabajo es que a mayor manipulación de las letras de Scrabble, mayor es el volumen de palabras generadas por los jugadores expertos. Los resultados confirmaron que, efectivamente, la acción física de la manipulación (Kirsh las llama epistémicas en Maglio y Kirsh, 1994) es una forma de utilizar interactivamente 
el entorno físico externo, lo que nosotros consideramos una forma de andamiaje, un proceso de simplificación cognitiva cercano al andamiaje para uno mismo de Mascolo.

Otro de los autores relevantes, especialmente para la relación de andamiaje con la cognición distribuida (Hutchins, 2004; Kirsh, 1995) y la mente extendida (Clark, 2004), es R. Pea (2004) al sugerir que debe hacerse una distinción entre dos maneras de organizar los apoyos a los procesos de aprendizaje. Una manera es un eje social que busca modelar la respuesta interactiva que depende contingentemente de las necesidades del individuo que aprende, permitiéndole realizar acciones que no podría llevar a cabo sin ayuda. La otra manera es un eje tecnológico, que gira en torno a los artefactos y su diseño. La tesis de Pea es que no deben confundirse estos dos ejes, y en particular, que el andamiaje para el aprendizaje entre personas no es acerca de los usos de artefactos tecnológicos, sino acerca de prácticas sociales que han surgido a lo largo de milenios (Pea, 2004, 430).

El concepto de affordance está ligado, inicialmente, a la obra de Gibson y es particularmente relevante su teoría de la percepción que expuso en su obra The ecological approach to visual perception, publicado en 1979. Según Gibson, la percepción es holística e integrada en un marco ecológico, de manera que las propiedades del entorno se perciben no como puntos diferentes y aislados sino como entidades significativas dentro de un determinado contexto ecológico de variables relacionadas entre sí. En este marco las affordances son relativas a la especie o grupo para el que tiene que facilitar la tarea (Gibson, 1979, 128). Por tanto, algo constituye una affordance no en términos absolutos sino en relación a un contexto determinado y para una especie determinada. En este sentido puede haber affordances que lo sean para los humanos y no para otras especies, y a la inversa.

También en el caso de affordance podemos seguir su evolución a través de distintos autores que han tomado esta idea de Gibson aplicándola a distintos campos. Lo que interesa es ver algunas de las caracterizaciones más relevantes, su adecuación o distanciamiento de la idea original de Gibson y las posibles semejanzas y diferencias.

Uno de los autores que ha utilizado el concepto de affordance es D. Norman, con algunas diferencias importantes respecto a Gibson. Para Gibson las affordances constituyen posibilidades de acción en el medio en relación a las capacidades de acción de un actor; son independientes de la experiencia y el conocimiento del actor; y tienen una existencia binaria, es decir, una affordance existe o no. Para Norman las affordances son propiedades percibidas que pueden existir o no en la realidad; constituyen sugerencias y claves para saber cómo utilizar las propiedades de un objeto; pueden depender de la experiencia, el conocimiento, o la cultura del actor; y pueden hacer que una acción sea fácil o difícil (McGrenere y Ho, 2000, 3). 
En la literatura sobre affordance también encontramos aproximaciones que nos proporcionan un panorama de los posibles sentidos de affordance. Especialmente interesante es el análisis de C.F. Michaels (2003), quien considera una serie de características relevantes de las affordances, señalando algunas diferencias con Gibson. Una de ellas es sobre la relación entre affordance y acción. Según Michaels, Gibson concibe que algo es una affordance si posibilita una acción, aunque no se lleve a cabo. Por ejemplo, una serpiente es una affordance para detectar el peligro, lo cual puede llevar a una acción, pero no necesariamente. Por tanto, indica una posible acción, pero no de forma automática. La postura de Michaels es que, si ampliamos tanto el concepto de affordance, éste queda diluido y puede llevar a confusión. Esto le lleva a relacionar affordances con "effectivities" (efectividades). La idea es que mientras las affordances constituyen propiedades del entorno que permiten a un animal realizar ciertas acciones, las efectividades serían las propiedades del animal que hacen posible realizar dicha acción. Si no encajan estas dos posibilidades la acción no puede llevarse a cabo. Michaels propone una relación más directa entre affordance y acción.

Según el filósofo de la percepción A. Noë (2004) la mirada, como experiencia visual, es una actividad de exploración que está mediada por nuestras habilidades sensorio- motrices. La mirada se convierte en un tipo de acción, como un gesto de las nadadoras o un movimiento en el agua. Noë, con una postura fenomenológica cercana a H. Dreyfus (1998), afirma: "Confiamos en que tenemos la habilidad de acceder a los detalles (de nuestra realidad física) con el movimiento, como base para nuestra sensación de presencia en el entorno como un todo (Noë, 2004, 51). La mirada, entonces, no tiene solamente un carácter individual, sino también está relacionada con las affordances de nuestro entorno físico y social.

Esta relación de affordances y acción tiene que ver con una de las ideas que subyacen a todo el tratamiento de las affordances, a saber: la interrelación entre percepción y acción. En uno de los que está explícito es en el artículo de Stroffregen et al. (2006). Estos autores argumentan que cuando decimos que la gente percibe affordances lo que percibe son posibles acciones que están disponibles en una determinada situación. En deporte, el concepto de affordance está siendo trabajado con éxito dentro de la perspectiva dinámica ecológica, encabezado por D. Araujo y colaboradores (Araujo et al., 2015). Dentro de la psicología del deporte, Araujo trabaja con una perspectiva externalista del proceso de entrenamiento que, en línia con el análisis que proponemos aquí, busca distanciarse del modelo individualista e internalista imperante dentro de esta disciplina.

\subsection{Recursos cognitivos para la estabilización del conocimiento}

La característica común de andamiaje y affordance es la de recurso cognitivo, pero para que sea eficaz no puede ser fugaz, ni siquiera pasajero. Se necesita poder estabilizar, al menos hasta cierto punto, dichos recursos. Y aquí es donde 
interviene la propuesta de R. F. Williams $(2004,5)$ sobre las formas de estabilizar el conocimiento que, en realidad, funcionan como soportes cognitivos. Williams se plantea la siguiente pregunta: "Dado nuestro limitado aparato conceptual, ¿cómo somos capaces de aprender conceptos complejos, para llevar a cabo procedimientos de cálculo complicados, y para mantener los logros cognitivos de nuestra especie en el tiempo, incluso construir sobre ellos, a través de múltiples generaciones?". La respuesta a esta cuestión es que los humanos disponemos distintas formas de superar estas limitaciones. Aquí Williams introduce tres formas o estructuras de estabilizar las conceptualizaciones, a saber: conceptual, material y social.

Primero, los recursos conceptuales incluyen diversas formas de organizar la información, por ejemplo, los "chunks" de Miller (1956) o los "frames" de Minsky (1975). También lo que Schank and Abelson (1977) llaman "scripts", como patrones de estructuras de acontecimientos, pueden considerarse recursos conceptuales, por ejemplo, cómo pedir comida en un restaurante o cómo asumir el rol de profesor. Todas estas formas de organizar la información podemos considerarlas como modelos conceptuales. Segundo, los recursos sociales son modelos conceptuales compartidos por los miembros de un mismo grupo, que D’Andrade (1989) denomina "modelo cultural". Son precisamente los modelos conceptuales y culturales los que permiten la comunicación y el entendimiento entre los humanos. Más específicamente, D’Andrade $(1995,45)$ afirma que la complejidad de la sociedad humana no sería posible sin la existencia de símbolos lingüísticos (palabras, pero también números) que nos permiten discriminar o agrupar la información en nuestra memoria de por sí precaria. En último término la base de los modelos culturales es simbólica y, a la vez, corpórea: según Williams (2004, 6), "Dado que los humanos tienen la misma configuración corporal y habitan el mismo mundo, los esquemas de imagen corpórea son universales para todos los seres humanos con un desarrollo normal". Es un recurso social en el sentido de que es la organización e interacción social la que hace posible intercambiar y compartir modelos conceptuales, pero al mismo tiempo vemos que ambos tipos de recursos están relacionados, ya que no es concebible un modelo cultural sin modelos conceptuales. Tercero, los recursos materiales son todas las huellas dejadas por los humanos que, en un momento determinado de la evolución, pudieron suponer la supervivencia y que ahora constituyen un ahorro cognitivo, si no para la supervivencia, sí para facilitar nuestra actividad como humanos. Ejemplos de recursos materiales hay muchos y de muy diversa índole, desde edificios y señales de tráfico, hasta la distribución de objetos en el espacio cotidiano. Además, los humanos construimos objetos y los introducimos en el entorno para facilitar la actividad cognitiva, a modo de memoria externa.

A fin de poner a prueba su propuesta Williams lo aplica a la actividad cognitiva de decir la hora (Daily Time-telling). La relevancia de esta actividad está en que intervienen los tres recursos mencionados: decir la hora implica que una persona interacciona con un artefacto (el reloj), lo cual requiere un modelo conceptual 
y un recurso material; además, aprender a decir la hora requiere instrucción, lo cual implica interacción social. En conclusión, es un caso de actividad cotidiana en la que confluyen recursos conceptuales, materiales y sociales, entrelazados pero distinguibles.

\subsection{Andamiajes y affordances como estabilizadores del conocimiento}

A partir de la cuestión planteada por Williams y D'Andrade sobre la complejidad cultural a la que la individualidad cognitiva y limitada de nuestra mente se tiene que enfrentar, planteamos aquí la necesidad de los procesos de estabilización para rutinizar lo aprendido y hacer posible la transmisión de conocimientos en la vida cotidiana y profesional. Por lo tanto, podemos analizar el rol de los andamiajes y affordances como recursos conceptuales, sociales y culturales como instrumentos de estabilización. Esta cuestión la podemos abordar en dos sentidos, por un lado, hasta qué punto andamiajes y affordances constituyen medios para estabilizar conocimiento (teórico y práctico); por otro, hasta qué punto los procesos de estabilización son la base para la efectividad de andamiajes y affordances a largo plazo. En último término, los recursos y la estabilización de los mismos son como las dos caras de una moneda. Supongamos que existe una affordance en el entorno que nos proporciona la oportunidad de poder subir la escalera, pero lo interesante es que este conocimiento perdure y que en otra ocasión parecida cuando veamos una escalera la percibamos como una affordance. Para que esto ocurra necesitamos que se estabilice este conocimiento. En sentido inverso, en los ejemplos de estabilización, podemos pensar que un andamiaje es una forma de estabilizar un conocimiento.

Teniendo en cuenta las diversas aproximaciones de andamiajes y affordances, podemos encontrar ejemplos de la interacción entre dichos conceptos y las formas de estabilización de Williams (2004). Así el eje social de Pea (2004) equivaldría a la forma social de estabilizar el conocimiento de Williams; y el eje tecnológico equivaldría a la forma material de estabilizar el conocimiento, siempre que lo material lo entendamos en sentido más amplio que lo tecnológico. Por tanto, podríamos decir que el eje tecnológico equivale a la forma material-tecnológica de estabilizar el conocimiento. Además, introducir un eje material no es incompatible con el eje social, ya que cualquier artefacto es un producto humano. De hecho, podemos encontrar en Pea $(2004,430)$ afirmaciones que van en la línea de una imbricación entre lo social y lo tecnológico: "El desafío teórico es que, para la especie humana, las herramientas y las tecnologías simbolizadoras (como el lenguaje escrito y los sistemas numéricos) se encuentran entre nuestros logros culturales más significativos", aunque, a veces, parece abogar por una distinción más radical. 


\section{Métodos}

Las observaciones se llevaron a cabo en la primavera de 2012, con 2 cámaras de vídeo. La negociación definitiva de entrada se inició en el Centro de Alto Rendimiento (CAR) del equipo olímpico espańol. Los autores estuvieron siguiendo al equipo en su día a día, observando cómo se distribuía el conocimiento, y cómo se tomaban decisiones. Tanto la entrenadora principal (Anna Tarrés) y la segunda entrenadora (Beth Fernández) como las nadadoras participaron en la observación y las entrevistas. El proceso de investigación se ha desarrollado en tres pasos: el acceso al campo, la recogida de los datos empíricos (notas y vídeo de campo) y el análisis y visualización de los resultados con el programa ELAN (figura 1). El programa ELAN fue desarrollado originalmente por el Instituto Max Planck de Psicolingüística para el análisis de micro-gestos e interacciones (Brugman y Husserl, 2004).

Los códigos de análisis se construyeron a través de lecturas, discusiones y conversaciones informales con los participantes (Corbin y Strauss, 1990). Definimos y determinamos un proceso estandarizado del entrenamiento que incluye los participantes, las herramientas o artefactos, las interacciones y/o acciones, y el contenido general de la interacción comunicativa del entrenamiento, como vemos en las figuras incluidas en el apartado de análisis. Schegloff (1996) enumera varias acciones que tienen lugar en la toma de turnos, como girar la cabeza hacia el futuro co-participante de la interacción, gesticular (Streeck y Hartge, 1992), sonreír, aspirar o simplemente, hablar. Mondada (2009) señala también que la fase de apertura de los encuentros conversacionales se logra cuando los participantes metódicamente movilizan una serie de recursos posibles: trayectorias y posturas corporales, miradas, y todo el elenco de actos verbales, como palabras, gritos e interjecciones. La mirada también se convierte en un tipo de acción, como un gesto de las nadadoras o un movimiento en el agua. 


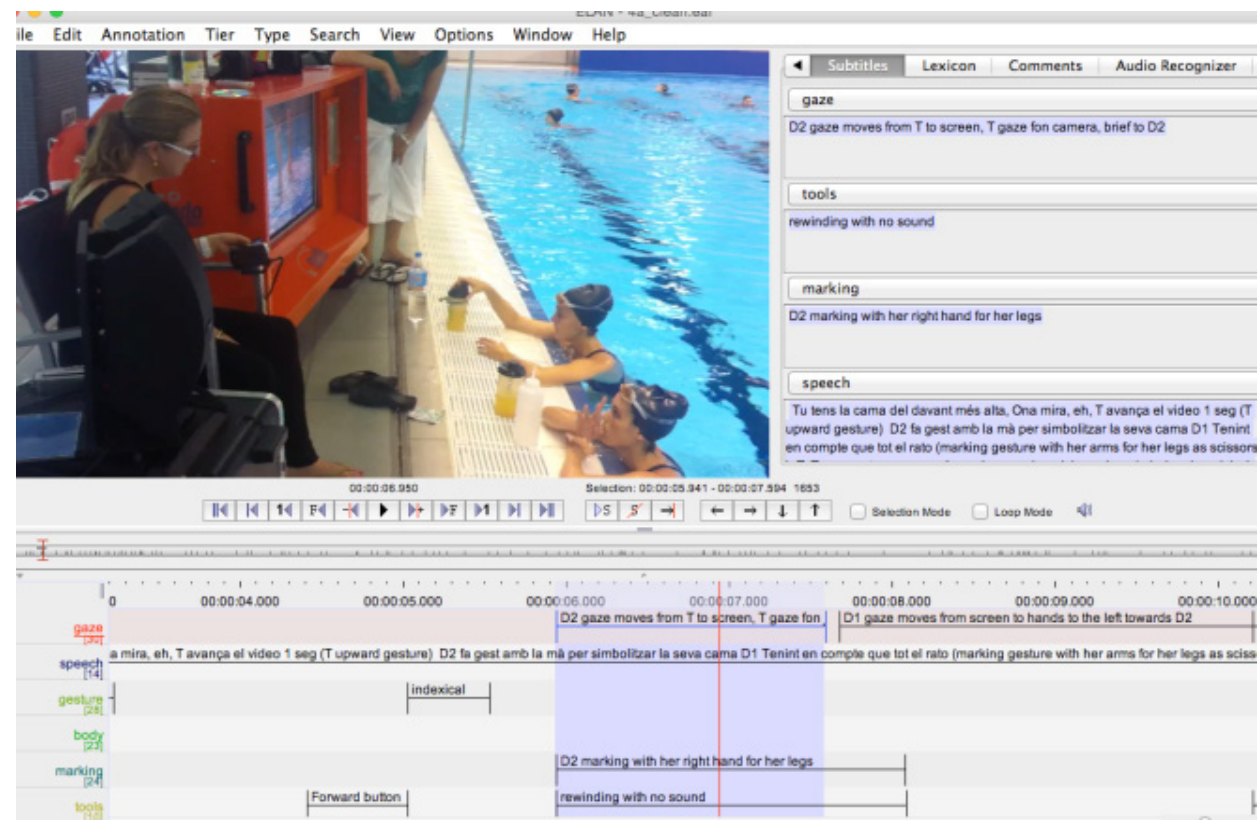

Figura 1. Análisis del proceso de entrenamiento de natación sincronizada, CAR, 2012. Anna Tarrés con Andrea Fuentes y Ona Carbonell.

\section{Resultados y discusión}

Desde una perspectiva cognitiva estos ensayos son muy relevantes, pues están a medio camino entre un proceso creativo y uno reproductivo. Siguiendo ideas de M. Csikszentmihalyi (2014) en buena parte de las disciplinas humanas podemos distinguir una fase creativa de una reproductiva. Por ejemplo, inventar una nueva coreografía, una nueva composición de piano o una nueva receta de arroz es un proceso creativo. Una vez la pieza musical, la receta o la coreografía están fijadas, los intérpretes básicamente la repiten. Es un proceso reproductivo.

En nuestro estudio nos encontramos con una combinación de ambas fases: no se trata simplemente de un proceso puramente creativo, como podría ser la entrenadora Anna Tarrés y las principales nadadoras imaginando posibles movimientos y cómo articularlos con la música que han decidido utilizar, ni tampoco un mero ejercicio de perfeccionar unos movimientos ya establecidos e inamovibles -que serían los ensayos finales- sino son unos ensayos prueba-error, en los que se observa si determinados movimientos imaginados realmente funcionan bien y son aplicables por las nadadoras, si estéticamente funcionan, si serán lo suficientemente interesantes como para permitir obtener una medalla en una competición, etc. En el momento de hacer el estudio las nadadoras principales que hicieron en Londres 2012, ganadoras de la medalla de plata, eran el dúo Andrea Fuentes y Ona Carbonell. Como grupo ganaron la medalla de bronce. 
En este proceso distribuido de redefinición de una coreografía observamos tres tipos de cambios y revisiones, confirmados después en entrevistas con entrenadoras y nadadoras:

i. Cambios directos ordenados por las entrenadoras al observar que algo no funciona o que es mejorable. Normalmente hay una negociación entre la entrenadora principal y la secundaria hasta que se toma la decisión. A veces las nadadoras principales son incluidas también en el debate.

ii. Cambios que surgen de observaciones directas de las nadadoras, son negociados con las entrenadoras y finalmente aceptados. Estos cambios se dan en un menor número que los descritos en i) pero pueden ser tan relevantes para la coreografía final como los tomados ahí.

iii. Cambios resultados de observar en los monitores de vídeo lo que las nadadoras han hecho (feedback). Hay un consenso básico entre nadadoras $\mathrm{y}$ entrenadoras sobre lo que ha funcionado y lo que no y entrenadoras o nadadoras proponen un cambio concreto para solucionar el problema. Los problemas detectados desde este mecanismo son básicamente de tres tipos: 1) hay un problema de sincronización entre nadadoras que requiere un mayor esfuerzo de focalización por parte de alguna de ellas, 2) cierta secuencia de movimientos no parece que pueda ser lograda por el equipo y debe ser abandonada, y 3) hay un gran salto visual entre cómo se imaginaban una escena y cómo resulta en realidad con lo que hay que replantear parte o la totalidad de la secuencia.

En términos de estabilización de recursos conceptuales, objetivo general de este artículo, procederemos a describir los elementos más relevantes de las pautas comunicativas de los entrenos observados para así establecer su función dentro de un esquema de andamiajes y affordances.

i. Un lenguaje gestual para referirse de forma relativamente precisa a gestos específicos de la coreografía (figura 2). Por ejemplo, levantar la mano y desplazar los dedos índice y medio para indicar un movimiento similar de las nadadoras mientras están cabeza abajo y desplazando las piernas con un movimiento similar.

ii. Los movimientos de las otras nadadoras que sirven como guía para recordar qué movimiento toca y observar si se está haciendo la coreografía tal y cómo debía ser (figura 3). 
iii. Monitores de vídeo al borde de la piscina para que las nadadoras puedan observarse después una vez acabado el ensayo de un grupo determinado de movimientos que se están redefiniendo (figura 4). Diversas cámaras de vídeo graban los movimientos de las nadadoras por encima y por debajo del agua y registran sus movimientos durante los ensayos. Las cámaras bajo el agua se usan normalmente asociados al trabajo biomecánico y tienen una función central en los ensayos que observamos para este artículo.

\subsection{El lenguaje gestual como dispositivo cognitivo}

¿Qué tipo de andamiaje son estos gestos que permiten a las nadadoras y entrenadoras referirse de forma clara y rápida a movimientos específicos? Siguiendo las ideas de Pea indicadas en la sección 1, este lenguaje gestual qua andamiaje es una práctica social y no solamente tecnológica. El uso de las manos para codificar movimientos y secuencias de una coreografía sería un andamiaje basado en prácticas sociales que vienen del mundo de la danza y se importaron en la natación sincronizada.

Observemos que no se trata de una mera asimilación de un lenguaje ajeno, sino que nos encontramos con un proceso de adaptación. La natación sincronizada incluye movimientos altamente improbables en danza clásica o contemporánea como puede ser estar cabeza abajo con los brazos pegados al cuerpo mientras las piernas se mueven frenéticamente hacia adelante y hacia atrás. De la misma forma, una buena parte del repertorio de la danza en suelo no tiene sentido en un medio acuático. 

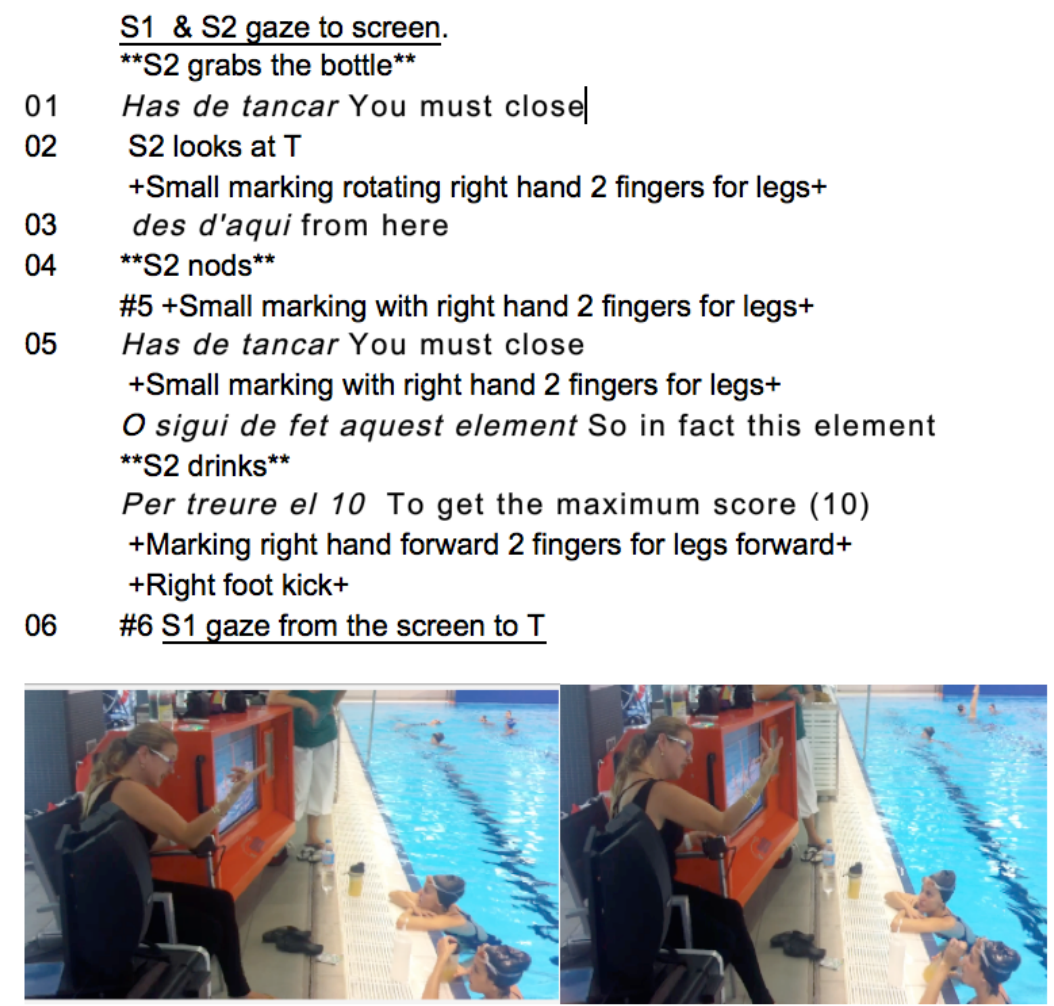

Figura 2. Ejemplo de gesto con contenido conceptual que constituye parte del andamiaje de la coreografía.

Este proceso de adaptación es posible al tratar también los gestos como affordances. La teoría más relevante aquí para entender estas affordances es más la original de Gibson (1979) que la revisada por Norman (1999). Así el gesto del dedo índice y medio moviéndose para indicar un desplazamiento no es convencional, sino un gesto natural que se entiende de manera intuitiva. Una prueba indirecta de ello es cómo los autores entendieron el gesto la primera vez que lo vieron en acción en la piscina, sin necesidad de que nadie del mundo de la natación sincronizada tuviera que explicárselo.

Si seguimos las ideas de Mascolo (2005), tenemos otro argumento a favor de un andamiaje social. Este lenguaje gestual deviene procesos mediante los cuales se producen intercambios con otras personas, en este caso, hacer referencia a movimientos concretos en una secuencia coreográfica. Secundariamente, podemos ver también aquí un caso de "personal scaffolding" en los que una nadadora individual utiliza los gestos para memorizar mejor una secuencia concreta. Es algo que pudimos observar varias veces en las nadadoras responsables del dúo, que antes de empezar un nuevo ensayo, flotando sobre el agua repetían una secuencia de gestos con las manos para recordar los movimientos que iban a desarrollar en el agua segundos después. 
Siguiendo las ideas de D’Andrade (1995) podríamos decir que el lenguaje gestual de movimientos coreográficos es un "modelo cultural". Y su raíz social se establece en un wittgensteniano "compartir una forma de vida" que presenta Williams (2004), tal y como queda explicado arriba, cuando observa que los esquemas corporizados son universales. Esta interacción entre modelo cultural y modelo conceptual, entre práctica social y naturaleza corporal es lo que hace que estos esquemas sean tan estabilizadores y funcionen tan bien como andamiajes cognitivos.

¿Cuál es la función del andamiaje? Facilitar el aprendizaje y la comprensión de las coreografías. Desde la perspectiva de Andy Clark (2004), nos encontramos así con el segundo sentido de andamiaje, la idea de "pensar con andamios": El andamiaje como ayuda crucial para el cerebro que, aunque potente, tiene sus limitaciones. Aunque se trate de un lenguaje, claramente no es el sentido 1 de Clark de andamiaje como instrumento conceptual que permita a las nadadoras nuevos pensamientos acerca de esos movimientos.

\subsection{La ayuda cognitiva de los movimientos de las compañeras}

Una coreografía en natación sincronizada tiene sentido al implicar la sincronización con los movimientos de como mínimo otra persona (dúo) o un grupo. Ese requisito se convierte también en una ayuda en los procesos de ensayo al convertirse los gestos de las demás nadadoras en un recordatorio y una guía de qué ha de hacer una nadadora específica.

Podrían considerarse así una affordance en el sentido de Norman. Aunque hay ciertos principios ecológicos y de materialidad específica asociado a cómo se desarrollan ciertas secuencias de movimientos (la naturaleza del agua y la estructura del cuerpo humano hace que ciertos movimientos sean naturales, otros extrańos, y otros directamente imposibles) el núcleo de una coreografía es resultado de una serie de convenciones: que haya que hundirse después de haber hecho una voltereta en la superficie es una convención establecida en el proceso creativo de la coreografía y ver cómo las compañeras empiezan a hacer el gesto de hundirse es una affordance estilo Norman que nos recuerda cuál es el siguiente movimiento que debemos hacer, estableciéndose así cierta interconexión entre percepción y acción (Wilson, 1977), Mountjoy, 1999). Dicho de otra forma, según las ideas de Stroffregen et al. (2006) "Affordances in the design of enactive systems" cuando decimos que las nadadoras y la entrenadora perciben affordances lo que percibe son posibles acciones (en nuestro caso movimientos coreografiados) que están disponibles en una determinada situación (en la piscina de entrenamiento). 
T Però si ella pot fer-ho, això, ho pot fer, a veure. But she can do it, this, she can do it, let's see

+Pointing twice+

Looking at $\mathrm{S} 1$

$08 \quad \frac{\text { S1 gaze from her } h a n d}{{ }^{*} \mathrm{~S} 2 \text { does it once }}{ }^{\star \star}$

T looking and talking to $S 1$, then both look at S2 in silence

+ Chin forward+

$09{ }^{* *}$ S2 does it again without getting out of the water **

$10 \quad \# 16$ S1 Es que encara carrega She is still charging

11 T Clar. Of course.

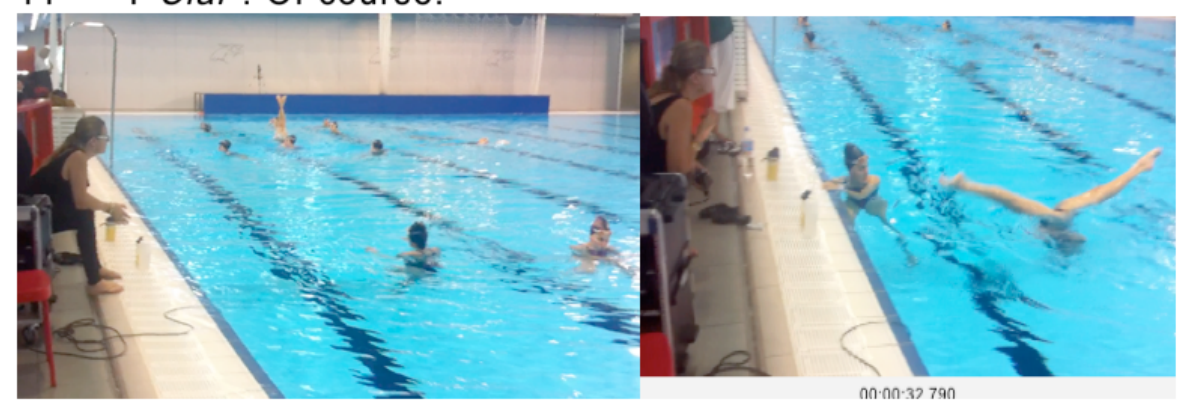

Figura 3. Los movimientos de las otras nadadoras del equipo con Anna Tarrés y Beth Fernández como andamiaje conceptual.

Los movimientos de las otras nadadoras invitan a la acción, mediante unas pautas de comunicación multimodal las nadadoras se miran las unas a las otras, como vemos en la figura 2 , pero también hablan entre ellas y con la entrenadora. La multiplicidad de modalidades utilizadas confirma la complejidad del proceso comunicativo. Precisamente, D’Andrade (1995) da un paso más y habla de la intersubjetividad de tales recursos cognitivos. El gesto de la nadadora es parte del tejido de significaciones de su equipo, de unos significados compartidos. Desde la cognición distribuida, se han definido estrategias de andamiaje parecidas en danza (Keevallik, 2010, Muntanyola-Saura y Kirsh, 2010, Muntanyola-Saura, 2015) con el nombre de marking (marcaje). Estos gestos vendrían a ser una estrategia cognitiva común a bailarinas y nadadoras, así como a los músicos y otros artistas que trabajan con el cuerpo. Permite que las nadadoras se comuniquen sin hacer el movimiento o la acción completa, mediante la selección parcial de aspectos tales como el peso, la velocidad, la dirección o la dinámica.

\subsection{El vídeo como andamiaje}

Las cámaras de vídeo son un instrumento central en el proceso de redefinición de una coreografía. La cámara de vídeo se ha convertido en un acompañante de cualquier deporte olímpico, pero en natación sincronizada es especialmente importante pues a diferencia de otros deportes terrestres, una parte relevante de 
las acciones que se están llevando a cabo no son prácticamente observables por las nadadoras al encontrarse bajo el agua. Así, cuando el equipo olímpico está cabeza abajo y agitando las piernas, las nadadoras no tienen otra forma de ver el efecto final ni si están realmente sincronizadas o no más que viendo el vídeo de su actuación. La aparición de la cámara de vídeo ha permitido la creación de coreografías complejas en natación sincronizada que habrían resultado bastante difíciles de completar sin su ayuda.

El vídeo es un recurso material de uso extensivo en este proceso de revisión y readaptación de coreografías. Cumple la función indicada de memoria externa, y es una parte natural del entorno. Cualquier equipo de natación sincronizada que aspire a conseguir algún hecho relevante dispone de un sistema mínimo de visualización y memorización en vídeo (Sydnor, 1998).

Es fácil ver que la cámara de vídeo actúa como andamiaje. Más específicamente como andamiaje en el sentido 3 de Clark, como "congelador del pensamiento". Gracias a la cámara, las nadadoras pueden reflexionar sobre su "performance" anterior y comprobar sus errores personales en movimiento y en coordinación.
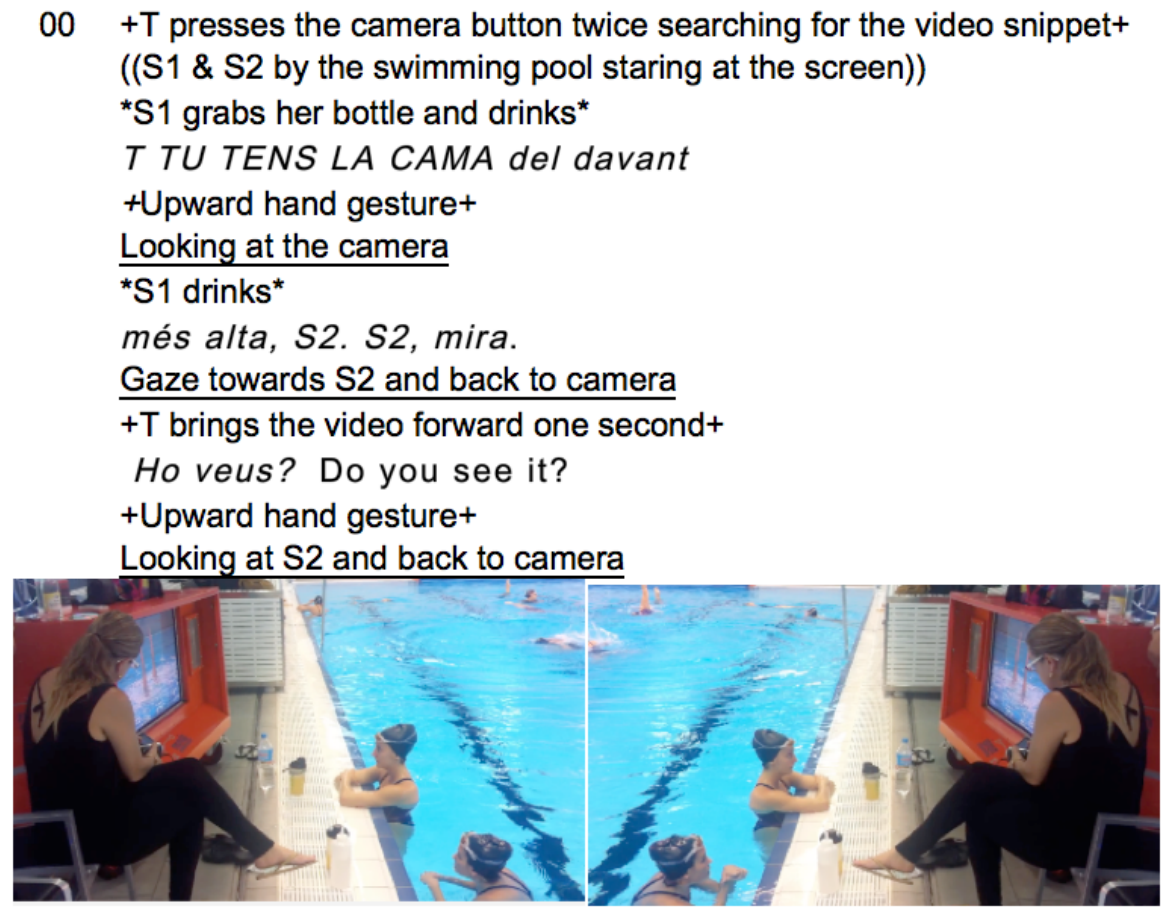

Figura 4. El vídeo y el monitor como artefactos cognitivos.

La manipulación de la cámara por parte de la entrenadora resulta una simplificación del proceso de memorización y de perfeccionamiento del proceso creativo. Este andamiaje parece encajar con la idea de proyección y simplificación de Magio y Kirsh (1995), que consideran las manipulaciones externas como andamiajes eficientes y prevalentes en el conocimiento experto. 


\section{4. ¿Qué hora es en la piscina?}

El entrenamiento de natación sincronizada es una actividad organizativa en el sentido de Noë (2015). En su recién publicado libro sobre estética y neurociencia, el filósofo rechaza la posibilidad de reducir la percepción y la actividad artística a un fenómeno psicológico como el de la percepción o la atención. El arte como actividad social escapa a las explicaciones causales de otros procesos como la fotosíntesis o la percepción de los colores. Cuando el equipo de natación entrena, entra en un proceso de investigación de su entorno, revisando sus creencias sobre cómo tiene que ser una coreografía y abriendo las puertas a nuevos movimientos. La sensibilidad artística, por lo tanto, tendría más en común con la actividad científica de investigación, o con el acto reflexivo de contemplarnos a nosotros mismos y al lugar que ocupamos en el mundo, que con un simple acto de percepción de una escalera de colores Pantone.

Si seguimos la forma en que Williams (2004) describe el proceso de dar la hora, veremos que sus ideas son fácilmente trasladables al mundo de la natación sincronizada. La diferencia central estaría en el hecho de que la acción de dar la hora es una actividad básicamente individual mientras que el proceso de redefinición de coreografías es una acción básicamente grupal. Observemos, en primer lugar, la importancia de una serie de recursos materiales: las cámaras de vídeo y los respectivos monitores. Las personas interactúan con estos recursos materiales desde un modelo conceptual específico que les permite ver errores, aciertos, cambios, mejoras donde nosotros no vemos más que una serie de nadadoras realizando movimientos peculiares en el agua. Este modelo conceptual se refuerza según se van observando movimientos de natación sincronizada, ayudando al aprendizaje de las nadadoras individuales.

Aquí entra la dimensión social, pues el aprendizaje de dar la hora es resultado no sólo de la interacción con el reloj, sino también con familia y educadores que nos explican todo el modelo conceptual asociado a dar la hora, así como las pautas metodológicas concretas para leer la hora en un reloj, etc.

Más específicamente, la propia coreografía es un modelo conceptual, que intenta capturar un evento concreto describiendo con detalle de qué forma se va a bailar en un campeonato específico. Vemos así la revisión del concepto a partir de las prácticas específicas capturadas por el vídeo y visualizadas por el monitor, siguiendo también el modelo de "herramienta extraña" de Nöe (2015). 


\section{Aplicaciones prácticas}

Gracias a los datos obtenidos por una etnografía cognitiva de unas sesiones de entrenamiento de natación sincronizada del equipo olímpico español, hemos podido comprender cómo contribuyen les andamiajes y affordances a la estabilización de los recursos cognitivos de la entrenadora y de las nadadoras.

Mediante un análisis conversacional de las interacciones comunicativas entre las entrenadoras y las nadadoras, hemos visto cómo la coreografía de la natación sincronizada se redefinía y transformaba en función de los problemas que iban apareciendo. Se han producido a veces divergencias muy relevantes que podían implicar transformar todo un grupo de movimientos, alterar la música original e incluso abandonar bloques supuestamente centrales de la coreografía. En lugar de un único núcleo decisorio, un ejercicio unidireccional en el que la entrenadora Anna Tarrés y su colaboradora Beth Fernández se encargarían de indicar cómo debían comportarse las nadadoras en todo momento, nos encontramos con un cambio un proceso de cognición distribuida, en el que las decisiones de revisar y transformar la coreografía eran resultado de un complejo proceso en el que ciertamente las entrenadoras tienen un papel central, pero en el que también participaban las nadadoras líder -responsables del dúo- las nadadoras del equipo, con un peso algo menor, y los datos ofrecidos por diferentes aparatos, especialmente las cámaras de vídeo, que eran, como hemos visto, determinantes.

Primero, hemos visto cómo el lenguaje gestual es dispositivo cognitivo en la línea de los andamiajes. Los gestos permiten tanto a las entrenadoras como a las nadadoras referirse de forma clara y rápida a movimientos específicos. Este lenguaje gestual qua andamiaje es una práctica social y no solamente tecnológica. El uso de las manos para codificar movimientos y secuencias de una coreografía sería un andamiaje basado en prácticas sociales que vienen del mundo de la danza y que probablemente se importaron en la natación sincronizada. Segundo, la multiplicidad de modalidades utilizadas confirma la complejidad del proceso comunicativo, que incluye el movimiento de las compañeras, una modalidad que podemos considerar de marcaje distribuido. Tercero, es fácil ver qué cámara de vídeo actúa como andamiaje. Más específicamente en el sentido que le da Clark (2004) de "congelador del pensamiento". Gracias a la cámara, las nadadoras pueden reflexionar sobre su performance anterior y comprobar sus errores personales en movimiento y en coordinación.

En definitiva, el proceso de revisión de una coreografía implica la interacción con un complicado entorno que incluye otros agentes sociales, recursos materiales, modelos culturales y conceptuales que se retroalimentan e influyen, pero que son finalmente distinguibles. 


\section{Referencias bibliográficas}

Araújo, D., Teques, P., Hernández-Mendo, A., Reiga, R. y Anguera. M. T. (2015). La toma de decisión, ¿es una conducta observable?: Discusión sobre diferentes perspectivas teóricas utilizadas en el estudio del rendimiento deportivo. Cuadernos de Psicología del Deporte, 16(1), 183-196.

Muntanyola-Saura, Dafne (2014). A cognitive account of expertise: Why Rational Choice Theory is (often) a Fiction. Theory y Psychology, 24, 19-39.

Muntanyola-Saura, Dafne (2015). Distributed Marking in Sport Corrections: A Conversation Analysis of Synchronized Swimming. Journal of Cognitive Science, 16(3), 338-354.

Muntanyola-Saura, Dafne y Kirsh, D. (2010). Marking as Physical Thinking: A Cognitive Ethnography of Dance. En L. A. Pérez Miranda y Aitor Izagirre Madariaga (Eds.), Proceedings of the IWCogSc-10 ILCLI International Workshop on Cognitive Science (pp. 339-355). Donosti: Universidad del País Vasco/Euskal Herriko Unibertsitatea-Servicio de Publicaciones.

Csikszentmihalyi, M. (2014). The Systems Model of Creativity. Dordrecht: Springer.

Clark, A. (1997). Being there: Putting body, brain, and world together again. Cambridge, Massachusetts: MIT Press.

D'Andrade, R. (1995). The Development of Cognitive Anhtropology. Cambridge, Massachusetts: MIT Press.

Cicourel, Aaron. (2006). The Interaction of discourse, cognition and culture. Discourse Studies, 8(1), 25-29.

Garfinkel, Harold. (1967). Studies in Ethnometodology. Los Angeles: Polity Press.

Gibson, J.J., (1979). The ecological approach to visual perception. Boston: Houghton Mifflin.

Goffman, Erwin. (1961). Asylums. New York: Anchor Books.

Hutchins, E. (1995a). Cognition in the wild. Cambridge, MA: MIT Press.

Hutchins, Ed. (2005). Material Anchors for Conceptual Blends. Journal of Pragmatics, 37, 10.

Keevallik, L. (2010). Bodily quoting in dance correction. Research on Language and Social Interaction, 43(4), 401-426.

Kirsh, D., y Maglio, P. (1994). On distinguishing epistemic from pragmatic action. Cognitive Science, 18, 513-549.

Kirsh, D. (2013). Embodied Cognition and the Magical Future of Interaction Design. ACM Transactions on Computer Human Interaction, 20, 1-30. 
Knorr-Cetina, Karin. (1999). Epistemic Cultures. Cambridge: Harvard UP.

Lozares, C. (ed.) (2007). Interacción, redes sociales y ciencias cognitivas. Granada: Comares.

Mascolo, M. F. (2005). Change processes in development: The concept of coactive scaffolding. New Ideas in Psychology, 23(3), 185-196.

Michaels, C. F. (2003). Affordances: Four points of debate. Ecological psychology, 15(2), 135-148.

Minsky, M. (1975). A framework for representing knowledge. En P. H. Winston (Ed.), The psychology of computer vision. New York: McGraw-Hill Book.

Mondada, L. (2009). Video recording practices and the reflexive constitution of the interactional order: some systematic uses of the split-screen technique. Human Studies, 32(1), 67-99.

Mountjoy, M. (1999). The basics of synchronized swimming and its injuries. Clinics in sports medicine, 18(2), 321-336.

Maglio, P. P., Matlock, T., Raphaely, D., Chernicky, B., y Kirsh D. (1999). Interactive skill in Scrabble. In Proceedings of Twenty-First Annual Conference of the Cognitive Science Society. Mahwah, NJ: Lawrence Erlbaum.

Noë, Alva (2004). Action in Perception. Cambridge, MA: MIT Press.

Noë, A. (2015). Strange Tools: Art and Human Nature. New York: Hill and Wang.

Norman, D. (1999). Affordance, conventions and design. Interactions, 6(3), 3843.

Pea, R. D. (2004). The social and technological dimensions of scaffolding and related theoretical concepts for learning, education, and human activity. The journal of the learning sciences, 13(3), 423-451.

Sacks, H., Schegloff, E. y Jefferson, G. (1978). A Simplest Systematic for the Organization of Turn-Taking of Conversation. En Jay Schenkein (Ed.), Studies in the Organization of Conversational Interaction. New York: Academic Press.

Smith, O. F. (2007). Object Artifact, Image Artifacts and Conceptual Artifacts: Beyond the object into the Event. Artifact, 1(1), 4-6.

Stoffregen, T. A., Bardy, B. G. y Mantel, B. (2006). Affordances in the design of enactive systems. Virtual Reality, 10(1), 4-10.

Sydnor, S. (1998). A history of synchronized swimming. Body Politics, 21, 252267. 
Vygotsky, Lev. (1978). Interaction between learning and development. En L. Vygotsky (Ed.), Mind in society (pp. 79-91). Cambridge: Harvard University Press.

Wacquant, Loic. (2004). Body and Soul: Notes of an Apprentice Boxer. New York: Oxford University Press.

Wilson, V. E. (1977). Objectivity and effect of order of appearance in judging of synchronized swimming meets. Perceptual and Motor Skills, 44(1), 295298.

Williams, Robert F. (2004). Making Meaning from a Clock: Material Artifacts and Conceptual Blending in Time-Telling Instruction. Ph.D. diss., University of California. 
